\title{
Estudo do papel do contexto linguístico no tratamento do desvio fonológico
}

\author{
Study of the role of the linguistic context in the treatment of \\ articulatory disorders
}

\author{
Giovana Ferreira Gonçalves ${ }^{1}$, Márcia Keske-Soares² ${ }^{2}$ Mardônia Alves Checalin $^{3}$
}

\begin{abstract}
RESUMO
Objetivo: Verificar a aplicabilidade dos ambientes favoráveis e neutros, obtidos a partir de dados de fala de crianças sem alteração de linguagem, no tratamento do desvio fonológico. Métodos: Seis crianças, com idade média de seis anos, com desvio fonológico, foram tratadas através do modelo ABAB - Retirada e Provas Múltiplas. As palavras selecionadas para o tratamento continham os ambientes favoráveis e neutros para a aquisição do /r/ segundo estudos de base teórica gerativa e gestual. A evolução terapêutica foi medida a partir dos dados das avaliações realizadas antes, durante e após o tratamento, sendo investigado, dentre os contextos utilizados, o mais eficaz para o tratamento. Resultados: O contexto neutro promoveu mais mudanças no sistema fonológico dos sujeitos do que o contexto favorável. Os dados dos sujeitos desse estudo apontaram contextos favoráveis para a aquisição do /r/ em crianças com desvio fonológico. Conclusão: O contexto linguístico favorável, baseado em dados de normalidade não é aplicável a crianças com desvio fonológico. Sugere-se a realização de pesquisas no tratamento do DF, utilizando os contextos propostos nesse estudo para testar sua aplicabilidade.
\end{abstract}

Descritores: Fala; Criança; Fonoterapia; Meio ambiente; Transtornos da articulação; Transtornos da linguagem

\section{INTRODUÇÃO}

A linguagem é o instrumento que utilizamos para transmitir as emoções e os pensamentos, ou seja, para comunicar, independente de ser expressa na forma oral, gestual ou corporal. A criança é exposta à linguagem desde muito cedo, ainda na vida intra-uterina, uma vez que a partir da vigésima semana de gestação o sistema auditivo já se encontra formado, apresentando ao novo ser o som do ambiente.

A fonologia corresponde ao modo como os sons da língua, os fonemas, se organizam e funcionam nas diferentes línguas faladas ao redor do mundo. Um estudo nacional ${ }^{(1)}$ considera que até a idade de cinco anos a criança deve conhecer e fazer o uso adequado dos fonemas presentes na língua materna.

Trabalho realizado na Universidade Federal de Santa Maria - UFSM - Santa Maria (RS), Brasil.

(1) Doutora, Professora do Curso de Graduação em Letras e dos Programas de Pós-Graduação em Letras e em Distúrbios da Comunicação Humana da Universidade Federal de Santa Maria - UFSM - Santa Maria (RS), Brasil.

(2) Doutora, Professora do Curso de Graduação em Fonoaudiologia e do Programa de Pós-Graduação em Distúrbios da Comunicação Humana da Universidade Federal de Santa Maria - UFSM - Santa Maria (RS), Brasil.

(3) Mestre, Fonoaudióloga clínica - Belém (PA), Brasil.

Endereço para correspondência: Mardônia Alves Checalin. R. Major

Lamarão, 2019, Bairro Val de Cans, Belém (PA), Brasil, CEP: 66115-690.

E-mail: mardoniafono@yahoo.com.br

Recebido em: 3/12/2008; Aceito em: 29/7/2009
O sistema fonológico é formado por classes de sons; dentre elas existe a classe das líquidas não-laterais, formadas pelos fonemas /r/ (caro) e /R/ (carro). Na prática clínica fonoaudiológica, comumente verifica-se que o /r/ é um dos últimos segmentos a ser adquirido pelas crianças em processo de terapia, surgindo daí o interesse pelo estudo sobre a aquisição do /r/ no tratamento de crianças com desvio fonológico (DF).

Quando a criança apresenta uma desorganização, inadaptação ou anormalidade no sistema fonológico em relação ao sistema padrão de sua comunidade linguística, sem que haja limitação articulatória, diz-se que tem $\mathrm{DF}^{(2)}$.

Crianças com DF apresentam uma desordem desenvolvimental diagnosticada quando a linguagem da criança é pobre em relação às outras habilidades sem razão aparente ${ }^{(3)}$; tal desordem acomete a produção dos sons da fala e varia de moderada, envolvendo poucos sons, a severa, envolvendo múltiplos erros na fala e baixa inteligibilidade ${ }^{(4)}$. As crianças com DF apresentam audição, inteligência nãoverbal e status neurológico normal com significante déficit na habilidade fonológica ${ }^{(5)}$.

Sabe-se que a terapia para DF baseada em um modelo terapêutico permite maior controle do tratamento e acompanhamento da evolução terapêutica. Atualmente, existem várias abordagens de terapia com base fonológica para o DF; contudo, neste estudo, a abordagem terapêutica 
escolhida, descrita na metodologia, foi o modelo ABAB Retirada e Provas Múltiplas ${ }^{(6)}$.

Outro ponto importante que a literatura destaca no tratamento do DF trata-se da seleção das palavras-alvo utilizadas em terapia. Há mais de uma década, autores ${ }^{(7)}$ valorizam a análise e manipulação criteriosa de alguns aspectos da palavra na qual o som-alvo está inserido, e citam como relevantes: a tonicidade da sílaba na qual o alvo se encontra, o som que precede e o que segue o alvo, o número de sílabas da palavra, a estrutura silábica e o fator semântico.

No processo de seleção das palavras-alvo, também tem sido considerado ambiente linguístico. Ambientes favoráveis significam contextos linguísticos facilitadores para a aquisição do fonema e contextos neutros são aqueles que não exercem um papel determinante na aquisição fonológica. Os contextos são analisados quanto à tonicidade da sílaba em que o fonema se encontra na palavra e quanto ao fonema precedente e seguinte ${ }^{(1)}$.

Estudos $^{(8-9)}$ baseados em diferentes abordagens teóricas apontam contextos facilitadores para a aquisição do $/ \mathrm{r} /$ quando esse segmento ocupa a posição silábica de onset medial (OM) - início de sílaba dentro da palavra - a (caro).

A Fonologia Gerativa propõe que a faculdade da linguagem, incluindo a aquisição fonológica, é um mecanismo inato. Um estudo ${ }^{(8)}$ baseado nessa perspectiva teórica traz como ambiente favorável o/r/ na sílaba tônica, antecedido por /i/ e seguido por $/ \mathrm{u} /$.

Já para a Fonologia Gestual, o gesto articulatório do segmento que antecede e sucede o alvo torna a propriedade acústico-articulatória mais previsível e natural. Em outras palavras, manobras articulatórias são adotadas como medida de economia em prol da fluência na pronúncia. Estudo ${ }^{(9)}$ baseado nessa perspectiva teórica traz, como contexto facilitador para $\mathrm{o} / \mathrm{r} /$, a sílaba tônica, antecedido por /a/ e seguido por /e/.

Esse estudo surgiu da necessidade de se promover intervenção terapêutica para crianças com DF mais rápida e eficaz, pois, apesar do vasto campo de atuação do fonoaudiólogo, este profissional é mais reconhecido como aquele que trabalha com crianças que falam errado. Isso eleva o número de encaminhamentos para atendimento nos serviços de fonoaudiologia públicos, resultando em filas de espera extensas.

Baseando-se na hipótese de que o tratamento com palavras que contemplam os ambientes favoráveis promoveria a aquisição do segmento mais precoce que o tratamento com palavras que contemplam ambientes neutros, o objetivo desse estudo foi avaliar e comparar os efeitos da terapia fonológica, utilizando palavras com ambientes favoráveis e neutros na emergência do /r/ no sistema fonológico de crianças com DF.

\section{MÉTODOS}

Foi realizada uma investigação preliminar acerca de contexto linguístico no DF, baseada no tratamento das alterações de fala de seis sujeitos com idade média de seis anos, com desvio fonológico, tratados através do modelo $\mathrm{ABAB}$ - Retirada e Provas Múltiplas.

A seleção dos sujeitos dessa pesquisa foi feita a partir de uma reunião com os responsáveis pelas crianças com alteração de fala que aguardavam atendimento na fila de espera de um serviço público de Fonoaudiologia. Na reunião, foi informada a existência do projeto de pesquisa de mestrado no qual seria realizado tratamento fonoaudiológico das alterações de fala dos sujeitos que se enquadrassem nos critérios exigidos pela pesquisa.

Mediante esclarecimento verbal e por escrito dos procedimentos da pesquisa, foi solicitada a autorização dos responsáveis para a participação das crianças através da assinatura do Termo de Consentimento Livre e Esclarecido aprovado pelo Comitê de Ética em Pesquisa da Universidade Federal de Santa Maria, registrado sob n ${ }^{\circ}$ 0182.0.243.000-07.

Antes de serem incluídas na pesquisa, as crianças passaram por uma bateria de avaliações fonoaudiológicas para verificar se atendiam às exigências da pesquisa.

Além dos exames complementares de avaliação audiológica, otorrinolaringológica e neurológica, foram realizados: avaliação fonológica obtida a partir nomeação de figuras baseadas em instrumento de avaliação ${ }^{(10-11)}$ e amostra de fala espontânea; exame articulatório; avaliação do sistema sensório-motor oral; avaliação do vocabulário; capacidade de discriminação auditiva; habilidades em consciência fonológica; habilidades em memória de trabalho e processamento auditivo.

Após as avaliações, fez-se a seleção das crianças que atendiam aos critérios de inclusão da pesquisa: idade superior a cinco anos, com DF e que apresentassem ausência, na avaliação fonológica, apenas do /r/ em no sistema fonológico.

Foi dado início ao tratamento da alteração de fala através do modelo ABAB - Retirada e Provas Múltiplas ${ }^{(6)}$. Os seis sujeitos selecionados tinham DF de grau médio ${ }^{(12)}$ e tiveram $\mathrm{o} / \mathrm{r} /$ tratado em onset medial (OM).

A aplicação do modelo teve início com a avaliação fonológica inicial (A1) que foi realizada através da nomeação de figuras ${ }^{(10-11)}$ e coleta de fala espontânea.

Após a avaliação, deu-se inicio ao ciclo de tratamento (B1) que teve duração de cinco semanas, nas quais foram realizadas nove sessões de terapia na frequência de duas sessões semanais de 45 minutos*.

Ao longo do ciclo de tratamento, mais especificamente na primeira, quinta e nona sessões de terapia, ocorreram as provas alvo básicas (PABs) que consistiam em sondagens do /r/ para verificar a evolução do som-alvo nas diferentes posições e estruturas silábicas. As figuras nomeadas nessa avaliação, em OM, foram, além das palavras-alvo, fogueira, xícara, girafa, urubu, tesoura e cenoura; em CM, foram garfo, ervilha, sorvete, borboleta, carta e urso; em CF, foram colher, ziper, tambor, regador, interruptor e liquidificador; $\mathrm{e}, \mathrm{em} \mathrm{OC}$, foram frango, broche, fritas, zebra, avestruz e presente.

No início e final de cada sessão de terapia, era lido um bombardeio auditivo constituído por uma lista contendo palavras com o $/ \mathrm{r} /$, ocupando a mesma posição silábica em que

* As palavras-alvo utilizadas em terapia serão descritas, no Quadro 1, juntamente com os dados de contexto. 
o segmento foi trabalhado durante o tratamento. A lista foi dada também aos pais para que fosse lida para a criança diariamente. A lista continha as seguintes palavras: pirulito, bombeiro, vara, aranha, coruja, mamadeira, coroa, cadeira, coração, buraco, areia, amora, caramelo, pare, fora, cara e sara.

Após o ciclo de tratamento, ocorreu o período de retirada (A2), no qual realizaram-se cinco avaliações fonológicas. Esse período tinha duração de três semanas, nas quais as cinco sessões de avaliação ocorriam na frequência de duas sessões semanais.

Essas avaliações correspondiam às provas de generalização (PGs), que eram realizadas mediante nomeação de figuras, e à coleta de fala espontânea. Ocorriam na seguinte ordem: duas provas de generalização (PGs), seguidas de coleta de fala espontânea, e de mais duas PGs.

Na primeira e na quarta PG, era aplicado o instrumento de avaliação fonológica ${ }^{(10-11)}$. Na segunda e na quinta PG, era solicitada a nomeação de figuras sorteadas de um jogo**, como se tratava de sorteio, a repetição dos estímulos geralmente não ocorria. A coleta de fala espontânea se dava na terceira sessão do período de retirada.

Ao final do período de retirada, na última $\mathrm{PG}$, eram verificados os percentuais de produção do fonema tratado e dos demais fonemas do sistema fonológico do sujeito para julgar a necessidade de continuar o tratamento ou não. Quando o fonema era produzido corretamente em, no mínimo, $80 \%$ das possibilidades, era considerado adquirido ${ }^{(13)}$. Caso contrário, era dado início a um novo ciclo de tratamento, mantendo-se o /r/ em OM como som-alvo.

As avaliações fonológicas foram gravadas em sala acusticamente tratada, utilizando-se um gravador digital da marca Panasonic ${ }^{\circledR}$ colocado a $10 \mathrm{~cm}$ da boca da criança, acoplado à camisa. Após o término das gravações, os dados de fala gravados foram transferidos para o computador, sendo realizadas as transcrições e análises contrastivas. Os dados de fala transcritos e as análises contrastivas foram conferidos por dois juízes com experiência em transcrição de dados de fala.

A aplicabilidade do modelo ABAB - Retirada e Provas Múltiplas foi evidenciada em estudos ${ }^{(12-17)}$ realizados com DF com diferentes graus de severidade. Esse modelo foi escolhido pelo fato de ser o mais adequado para a proposta do estudo, pois a seleção de um som-alvo para o tratamento permitiu o controle do contexto linguístico.

As palavras-alvo utilizadas no tratamento contemplavam o contexto linguístico favorável, baseado na Fonologia Gerativa $^{(8)}$ e na Fonologia Gestual ${ }^{(9)}$, e o contexto neutro. O Quadro 1 destaca os contextos trazidos pelas abordagens teóricas e as palavras-alvo selecionadas para o tratamento.

Para a análise dos dados, dividiram-se os seis sujeitos em três grupos: contexto favorável gerativo, contexto favorável gestual e contexto neutro, com dois sujeitos em cada. Após isso, fez-se a comparação do desempenho**** dos sujeitos ao longo do tratamento através do percentual de realização do /r/ em OM na avaliação inicial, nas avaliações realizadas ao longo do tratamento, PABs, e nas avaliações realizadas após os ciclos de tratamento.

A análise dos dados foi realizada de modo qualitativo, observando-se o momento em que ocorreria a aquisição do segmento e existência uma abordagem teórica mais favorecedora para a aquisição. Considerando que o segmento estava adquirido quando apresentava percentual de realização igual ou superior a $80 \%^{(13)}$.

\section{RESULTADOS}

Todos os seis sujeitos apresentavam, na avaliação fonológica inicial, percentual de produção do /r/, em OM, inferior a $16 \%$, sendo iniciado o primeiro ciclo de tratamento com a PAB 1.

A Figura 1 torna possível evidenciar a evolução terapêutica dos seis sujeitos ao longo do primeiro ciclo de tratamento. Apenas S1 apresentou sinais de aquisição do /r/ durante o primeiro ciclo de tratamento, comprovados pelo percentual de realização do segmento nas PABs 2 e 3 (100\%). S4 e S6 apresentaram melhora no percentual de realização do segmento, porém, não atingiam percentuais característicos de fonema adquirido. S5 não mostrou evolução. E S2 e S3 apresentaram redução do percentual de produção do segmento em relação à $\mathrm{AF}$ inicial.

A gradualidade na aquisição é observada em S4 e S6, uma vez que o percentual de realização do /r/ foi aumentando ao longo das PABs. A regressão é perceptível no sistema de S2 e

Quadro 1. Contextos e palavras-alvo adotadas neste estudo

\begin{tabular}{|c|c|c|c|c|c|}
\hline Sujeitos & Abordagem teórica & Contex & & Palavras & alvo \\
\hline $\mathrm{S} 1$ e $\mathrm{S} 2$ & Gerativo favorável & $\begin{array}{l}/ \mathrm{i} / \neg \mathrm{r} \\
\quad \downarrow \\
\text { tônica }\end{array}$ & $\begin{array}{l}\quad r \rightarrow / u / \\
\downarrow \\
\text { tônica }\end{array}$ & $\begin{array}{l}\text { Siri } \\
\text { Irado } \\
\text { Pirata }\end{array}$ & $\begin{array}{l}\text { Peru } \\
\text { Peruca } \\
\text { Marujo }\end{array}$ \\
\hline S3 e S4 & Gestual favorável & $\begin{array}{r}/ \mathrm{a} / \neg \mathrm{r} \\
\quad \downarrow \\
\text { tônica }\end{array}$ & $\begin{array}{l}\quad r \rightarrow / e / \\
\downarrow \\
\text { tônica }\end{array}$ & $\begin{array}{l}\text { Marido } \\
\text { Barata } \\
\text { Farinha }\end{array}$ & $\begin{array}{l}\text { Careta } \\
\text { Cereja } \\
\text { Parede }\end{array}$ \\
\hline S5 e S6 & Neutro & $\begin{array}{r}/ \mathrm{o} / \neg \mathrm{r} \\
\downarrow \\
\text { tônica }\end{array}$ & $\begin{array}{c}r \rightarrow / E / \\
\downarrow \\
\text { tônica }\end{array}$ & $\begin{array}{l}\text { Chorão } \\
\text { Morango } \\
\text { Sorine }\end{array}$ & $\begin{array}{l}\text { Careca } \\
\text { Farelo } \\
\text { Amarelo }\end{array}$ \\
\hline
\end{tabular}

\footnotetext{
** Tratava-se do Jogo Lince, no qual a criança deveria procurar rapidamente no tabuleiro a figura sorteada em um saco plástico opaco.

*** Apesar de as avaliações fonológicas terem ocorrido em sala acusticamente tratada, em uma primeira análise, os resultados aqui apresentados foram obtidos a partir de uma análise de oitiva, ou seja, perceptivo-auditiva, em razão do tempo disponível para a conclusão do trabalho.
} 


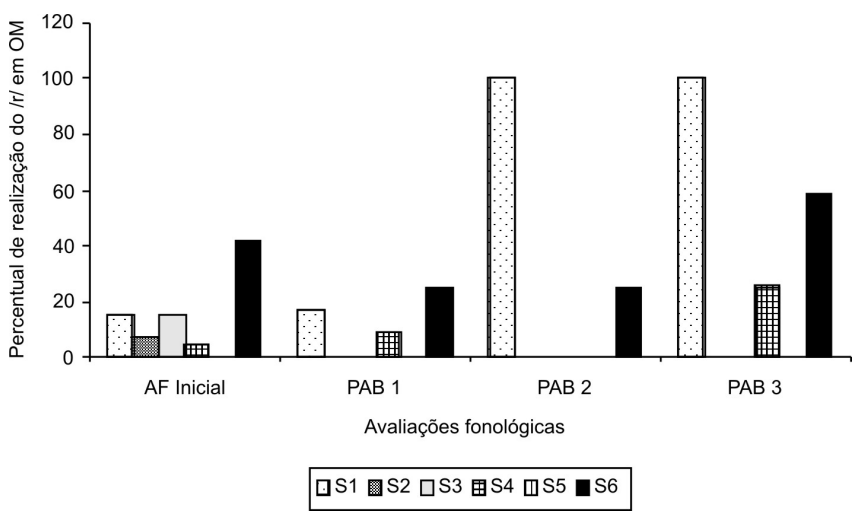

Legenda: $A F=$ avaliação fonológica; $P A B=$ prova alvo básica; $O M$ = onset medial

Figura 1. Primeiro ciclo de tratamento

$\mathrm{S} 3$, pois o percentual de produção do segmento caiu para $0 \%$ em ambos os casos.

As avaliações fonológicas realizadas durante o primeiro período de retirada confirmaram a aquisição do /r/ no sistema fonológico de S1. Apresentaram progressão no percentual de produção do /r/ S2, S3 e S5. As avaliações de S4 e S6 evidenciaram um sistema instável, devido à variação no percentual de realização do /r/ ao longo das provas, como pode ser constatado na Tabela 1.

Os dados de S6 apresentaram-se bastante instáveis, estando o /r/ adquirido, com percentual de produção superior a $80 \%$, em três das cinco provas realizadas no primeiro período de retirada. Essa característica foi considerada na decisão de se realizar mais um ciclo de tratamento com /r/ em OM em S6.

Foi dada continuidade ao tratamento, iniciando-se o segundo ciclo com todos os sujeitos, exceto com S1. A Figura

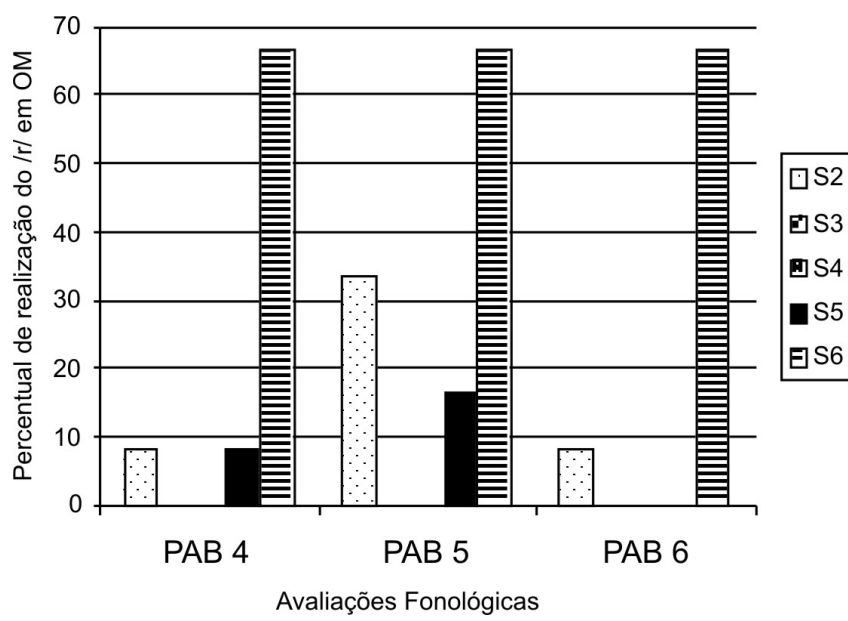

Legenda: $\mathrm{S}$ = sujeito; $\mathrm{PAB}$ = prova alvo básica; $\mathrm{OM}=$ onset medial

Figura 2. Segundo ciclo de tratamento

2 traz o percentual de realização do /r/ durante o segundo ciclo de tratamento.

É possível observar que, no segundo ciclo de tratamento, assim como no primeiro, S6 apresenta uma evolução terapêutica melhor que os demais. A Figura 2 mostra a estabilidade no sistema de S3, S4 e S6, não ocorrendo variação no percentual de produção do /r/. Em S2 e S5, a melhora no percentual de produção do /r/ na PAB 5, seguida de regressão na PAB 6, marcou a não-linearidade.

A Tabela 2 traz o percentual de realização do /r/ após o segundo ciclo de tratamento. Ocorre instabilidade do /r/ no sistema fonológico de quatro dos cinco sujeitos ainda em tratamento. As avaliações evidenciam a aquisição do /r/ em OM por S6 e melhora no percentual de produção por todos os sujeitos, exceto em S4.

Após o segundo ciclo, nas três últimas provas, S6, tratado

Tabela 1. Realização do /r/ em OM após o primeiro ciclo

\begin{tabular}{lccccc}
\hline & $P G 1\left(1^{a}\right.$ sessão $)$ & $P G 1\left(2^{a}\right.$ sessão $)$ & FE & $P G 2\left(1^{\text {a }}\right.$ sessão $)$ & PG 2 (2 ${ }^{\mathrm{a}}$ sessão $)$ \\
\hline S1 & 100 & 100 & - & 100 & 100 \\
S2 & 0 & 0 & 0 & 14,28 & 29,41 \\
S3 & 0 & 0 & 0 & 0 & 8,33 \\
S4 & 0 & 5,55 & 0 & 7,14 & 0 \\
S5 & 0 & 0 & 0 & 0 & 3,33 \\
S6 & 92,3 & 68,42 & 83,33 & 81,81 & 57,14 \\
\hline
\end{tabular}

Legenda: $\mathrm{S}=$ sujeito; $\mathrm{PG}$ = prova de generalização; $\mathrm{FE}=$ fala espontânea; $\mathrm{OM}=$ onset medial Valores expressos em \%

Tabela 2. Realização do /r/ em onset medial após o segundo ciclo

\begin{tabular}{|c|c|c|c|c|c|}
\hline & PG 3 ( $1^{a}$ sessão) & PG 3 (2a sessão) & FE & PG 4 (1 ${ }^{a}$ sessão) & PG 4 ( $2^{a}$ sessão) \\
\hline S2 & 0 & 7,14 & 25 & 5,88 & 8,33 \\
\hline S3 & 7,14 & 33,33 & 16,67 & 38,46 & 53,84 \\
\hline S4 & 0 & 0 & 0 & 0 & 0 \\
\hline S5 & 25 & 0 & 100 & 57 & 27,77 \\
\hline S6 & 87,5 & 75 & 100 & 100 & 90,47 \\
\hline
\end{tabular}

Legenda: $\mathrm{PG}$ = prova de generalização; $\mathrm{FE}$ = fala espontânea; $\mathrm{S}$ = sujeito

Valores expressos em \% 
Tabela 3. Realização do /r/ em onset medial após o terceiro ciclo

\begin{tabular}{lccccc}
\hline & $P G 5\left(1^{\text {a }}\right.$ sessão $)$ & $P G 5\left(2^{a}\right.$ sessão $)$ & FE & $P G 6\left(1^{a}\right.$ sessão $)$ & $P G 6\left(2^{a}\right.$ sessão $)$ \\
\hline S2 & 73,33 & 75 & 77,77 & 59,09 & 100 \\
S3 & 100 & 92,85 & 100 & 100 & 94,44 \\
S4 & 6,67 & 0 & 0 & 0 & 0 \\
\hline
\end{tabular}

Legenda: $P G$ = prova de generalização; $F E$ = fala espontânea

Valores expressos em \%

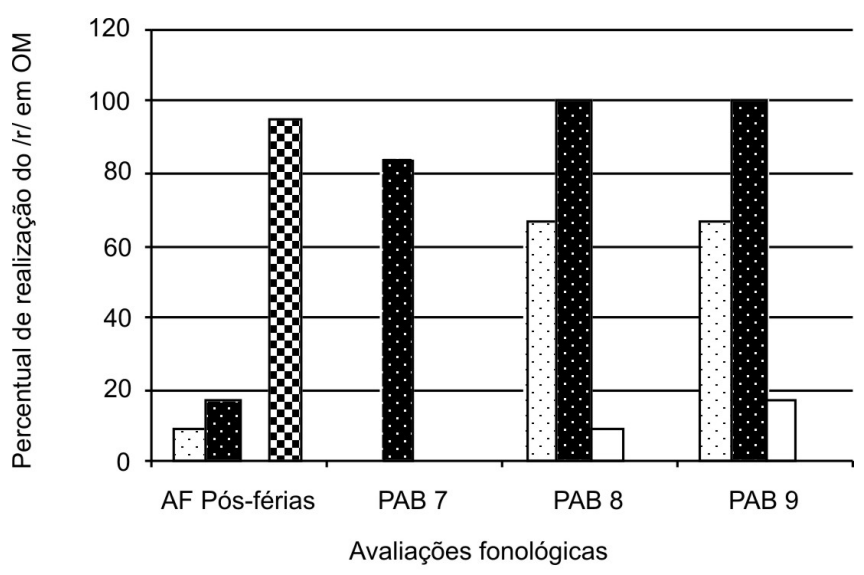

口S2 IS3 QS4 QS5

Legenda: $A F$ = avaliação fonológica; $\mathrm{PAB}$ = prova alvo básica; $\mathrm{OM}$ = onset medial

Figura 3. Terceiro ciclo de tratamento

por um contexto neutro, adquiriu o/r/ em OM. Os demais continuaram em tratamento uma vez que $\mathrm{o} / \mathrm{r} /$ ainda não havia sido adquirido ao final do segundo período de retirada. S4 foi o único que não apresentou evolução ao longo do segundo ciclo, ao contrário dos demais sujeitos.

O fato do ciclo do modelo ABAB - Retirada e Provas Múltiplas ser realizado em quatorze semanas, conforme explicado na metodologia, tornou necessária a realização de uma pausa no tratamento dos sujeitos para as festividades de final de ano. Ao retomar o tratamento, antes de iniciar o terceiro ciclo, foi feita uma avaliação fonológica para sondar o sistema fonológico da criança após o recesso.

A Figura 3 traz a avaliação fonológica pós-férias e aquelas realizadas ao longo do terceiro ciclo dos sujeitos. S5 apresentou o/r/ adquirido em OM após as férias com realização de 19 dos 20 itens lexicais sondados, correspondendo a $95 \%$ de realização correta do /r/ em OM. Seguiram em tratamento apenas três dos seis sujeitos que iniciaram: S2, S3 e S4.

$\mathrm{O}$ terceiro ciclo de tratamento promoveu a aquisição do segmento em S3, uma melhora efetiva no percentual de produção em S2 e uma melhora discreta em S4 (Figura 3).

Como pode ser visto na Tabela 3, após o terceiro ciclo, realizou-se o terceiro período de retirada, o qual comprovou a aquisição do/r/ por S2 e S3. S4 manteve-se com percentuais zerados de produção em praticamente todas as provas.

Fez-se o levantamento dos itens lexicais com /r/ em OM produzidos corretamente pelos sujeitos durante seus tratamentos a fim se comparar os contextos linguísticos mais frequentes com aqueles utilizados nesse estudo.

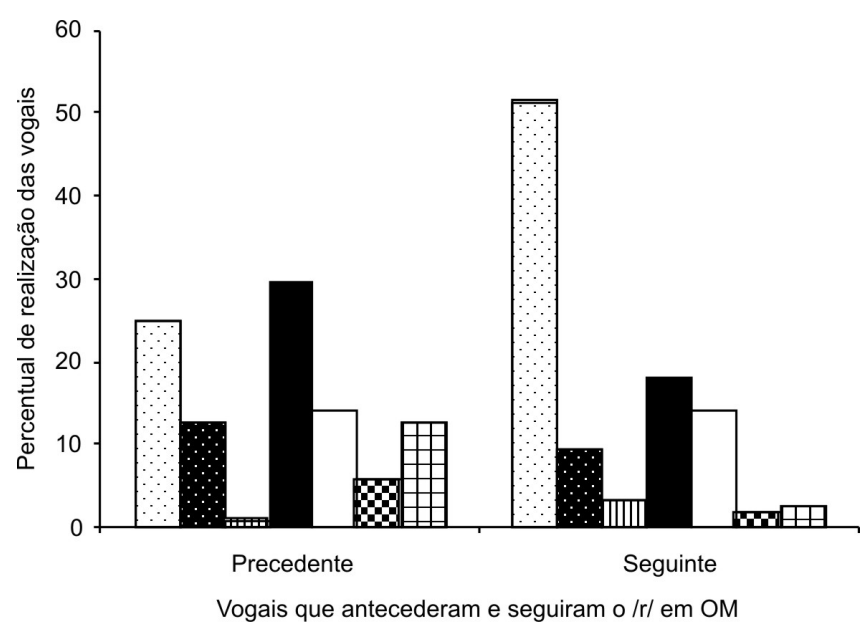

$\square$ ロ

Legenda: $\mathrm{DF}$ = desvio fonológico; $\mathrm{OM}$ = onset medial

Figura 4. Contexto linguístico mais frequente no tratamento do DF

A vogal /i/ foi o contexto precedente mais frequente, com $29,75 \%$ de ocorrência nas palavras produzidas pelos sujeitos em tratamento, assim como a vogal /a/ foi o contexto seguinte mais frequente, com $51,24 \%$ de ocorrência. Em relação ao contexto tonicidade, $49,59 \%$ das palavras produzidas traziam o /r/ em sílaba tônica (Figura 4).

\section{DISCUSSÃO}

Inicialmente foram desenvolvidas pesquisas em contexto linguístico na aquisição fonológica normal do Português Brasileiro e, recentemente, tem-se estudado o papel do contexto linguístico baseado na aquisição fonológica normal no tratamento do desvio fonológico ${ }^{(18)}$.

Dois estudos $^{(8,19)}$ desenvolvidos com dados de aquisição fonológica normal e um terceiro ${ }^{(18)}$ foi desenvolvido a partir de dados de uma criança com DF trouxeram contextos linguísticos facilitadores distintos. Os estudos supracitados apontaram, respectivamente, como contexto antecedente facilitador as vogais /i/, /i/, /a/; como contexto seguinte facilitador as vogais /u/, /i/, /a/; concordando, apenas, com o fato da sílaba tônica ser mais favorecedora à aquisição segmental. A divergência observada em relação aos contextos trazidos por esses estudos despertou o interesse em pesquisar contextos linguísticos facilitadores para a aquisição fonológica no tratamento do DF.

No presente estudo, foram controladas as variáveis linguísticas (contextos antecedente, seguinte e tonicidade) facilitadoras e neutras, segundo estudos baseados em diferentes 
abordagens teóricas, nas palavras-alvo utilizadas no tratamento de seis sujeitos.

$\mathrm{S} 1$ adquiriu o segmento-alvo em um ciclo de tratamento; $\mathrm{S} 5$ e S6 adquiriram o /r/ em OM em dois ciclos; S2 e S3 adquiriram o /r/ em três ciclos; e S4 não adquiriu ao final do terceiro ciclo S1, tratado por um contexto favorável, com base em pesquisa em Fonologia Gerativa, foi o primeiro a adquirir o /r/ em OM, durante o primeiro ciclo, seguido dos sujeitos tratados pelo contexto neutro, S5 e S6, durante o segundo ciclo de tratamento. S3, tratado por um contexto favorável baseado na Fonologia Gestual, e S2, tratado com contexto favorável baseado na Fonologia Gerativa, adquiriram o segmento no terceiro ciclo. E S4, tratado pelo contexto favorável baseado na Fonologia Gestual, não adquiriu o segmento com três ciclos de tratamento.

Embora S1 tenha adquirido o/r/ em um ciclo de tratamento, não é possível afirmar que o contexto baseado na Fonologia Gerativa promova aquisição do segmento mais rápida, pois $\mathrm{S} 2$, o outro sujeito que constitui o grupo, adquiriu o/r/ no terceiro ciclo, indicando que o resultado do tratamento de S1 trata-se de um evento isolado.

Os dados indicam que o contexto neutro foi mais eficaz no tratamento do /r/ em OM, pois os dois sujeitos do grupo, S5 e S6, adquiriram o segmento no segundo ciclo. Por se tratar de um assunto novo, não foi encontrada na literatura pesquisa semelhante que possibilitasse a comparação dos resultados.

$\mathrm{O}$ contexto linguístico mais frequente nas produções corretas de /r/ realizadas pelos sujeitos em tratamento para $\mathrm{DF}$, apresentados em um estudo ${ }^{(18)}$, também não coincidiram com o contexto facilitador indicado nas pesquisas em aquisição fonológica normal.

Fazendo-se um levantamento dos itens lexicais realizados corretamente pelos sujeitos durante o tratamento, os dados apontaram a vogal /i/ $(29,75 \%)$ e a vogal /a/ $(24,79 \%)$, como contextos precedentes mais frequentes, concordando com os estudos ${ }^{(8,19)}$ baseados em dados de aquisição normal que indicam a vogal /i/ como facilitadora para a aquisição e com o estudo ${ }^{(18)}$ baseado em dados de fala desviante que indicam a vogal /a/ como mais frequente.

Em relação ao contexto seguinte, esse estudo aponta a vogal /a/ como a mais frequente, ocorrendo $51,24 \%$ nas palavras produzidas corretamente, concordando, apenas, com o estudo ${ }^{(18)}$ baseado em dados de fala de crianças com DF no qual vogal /a/ foi o contexto seguinte mais frequente nas produções corretas. Um estudo anterior ${ }^{(8)}$ aponta a vogal /u/ como contexto seguinte facilitador e outro estudo ${ }^{(19)}$ aponta a vogal /i/ como contexto seguinte facilitador para a aquisição do /r/ em OM. Isso indica que o contexto linguístico favorável na aquisição fonológica normal não coincide com o contexto favorável no tratamento do DF.

Os estudos considerados ${ }^{(8,18-19)}$ são unânimes em afirmar que a sílaba tônica é mais favorecedora para a aquisição do $/ \mathrm{r} /$, concordando com os achados dessa pesquisa.

\section{CONCLUSÃO}

Os dados desse estudo indicaram que o contexto neutro, baseado em dados de normalidade, foi mais eficaz no tratamento do /r/ em OM que o contexto favorável.

$\mathrm{Na}$ investigação dos contextos linguísticos mais frequentes nos dados de fala das crianças que participaram desse estudo, surgiram resultados diferentes daqueles apontados nas pesquisas em aquisição normal, reforçando a hipótese de que os dados de contexto baseados na aquisição fonológica normal não são aplicáveis ao tratamento do DF.

O cuidado na seleção das palavras-alvo utilizadas no tratamento do DF é de fundamental importância para o sucesso terapêutico. Esse estudo acrescenta uma nova luz a esse processo, ou seja, aponta para a importância de considerar o contexto linguístico na seleção das palavras utilizadas em terapia.

Para isso, é importante a realização de mais pesquisas acerca do contexto linguístico na população com aquisição fonológica desviante para se chegar a resultados mais consistentes, a fim de se potencializar o tratamento, favorecendo a uma aquisição segmental mais rápida.

Infelizmente, ainda são poucas as pesquisas realizadas com contexto linguístico facilitador para a aquisição segmental em crianças com DF falantes do Português Brasileiro. É interessante a realização de mais estudos em contexto linguístico de modo a se encontrar resultados sólidos que promovam a aquisição segmental mais rápida no processo terapêutico.

\begin{abstract}
Purpose: To verify the applicability of favorable and neutral environment, obtained from speech data of children without language impairment, in the treatment of phonological disorder. Methods: Six children with phonological disorder, with an average age of six years, were treated using the ABAB - Withdrawal and Multiple Probes model. The words selected for treatment contained neutral and favorable environments for the acquisition of / $\mathrm{r} /$ according to gestural and generative theoretical basis studies. The therapeutic evolution was measured based on the data of the assessments carried out before, during and after the treatment, and investigated, among the contexts used, the most effective for treatment. Results: The neutral environment promoted more changes in the phonological system of the subjects than the favorable environment. The data of the subjects of this study showed favorable contexts for the acquisition of $/ \mathrm{r} /$ in children with phonological disorders. Conclusion: The favorable linguistic context, based on normality data, is not applicable to children with phonological disorders. It is suggested the realization of research in the treatment of phonological disorders using the contexts proposed in this study to test their applicability.
\end{abstract}

Keywords: Speech; Child; Speech therapy; Environment; Articulation disorders; Language disorders 


\section{REFERÊNCIAS}

1. Lamprecht RR, Bonilha G, Freitas C. Matzenauer C, Mezzomo C, Oliveira. Aquisição fonológica do português: perfil de desenvolvimento e subsídios para a terapia. Porto Alegre: Artmed; 2004.

2. Grunwell P . Os desvios fonológicos numa perspectiva linguística. In: Yavas M, organizador. Desvios fonológicos em crianças: teoria, pesquisa e tratamento. Porto Alegre: Mercado Aberto; 1990.

3. Bishop DV, Hayiou-Thomas ME. Heritability of specific language impairment depends on diagnostic criteria. Genes Brain Behav. 2008;7(3):365-72.

4. Sices L, Taylor HG, Freebairn L, Hansen A, Lewis B. Relationship between speech-sound disorders and early literacy skills in preschoolage children: impact of comorbid language impairment. J Dev Behav Pediatr. 2007;28(6):438-47.

5. Pawłowska M, Leonard LB, Camarata SM, Brown B, Camarata MN. Factors accounting for the ability of children with SLI to learn agreement morphemes in intervention. J Child Lang. 2008;35(1):25-53.

6. Tyler A, Figursky GR. Phonetic inventory changes after treating distinctions along an implicational hierarchy. Clin Linguist Phon. 1994. 8(2):91-107.

7. Lowe RJ, Weitz JM Intervenção. In: Lowe RJ. Fonologia: avaliação e intervenção: aplicação na patologia da fala. Porto Alegre: Artes Médicas; 1996.

8. Miranda AR. A aquisição do "r": uma contribuição à discussão sobre seu status fonológico [dissertação]. Porto Alegre: Pontifícia Universidade Católica do Rio Grande do Sul, Faculdade de Letras; 1996.

9. Albano EC. Sobre o abrimento 3 de Mattoso Câmara: pistas fonotáticas para a classe das líquidas. Estudos da Língua(gem). 2005; (2): 45-66.
10. Yavas M., Hernadorena CM, Lamprecht RR. Avaliação fonológica da criança: reeducação e terapia. Porto Alegre: Artes Médicas; 1991.

11. Matzenauer-Hernandorena CL, Lamprecht RR. A aquisição das consoantes líquidas do português. Letras de Hoje. 1997; 32(4):7-22.

12. Shriberg LD, Kwiatkowski J. Phonological disorders I: a diagnostic classification system. J Speech Hear Disord. 1982; 47(3):226-41.

13. Bernhardt B. Developmental implications of nonlinear phonological theory.Clin Linguist Phon. 1992; 6(4):259-81.

14. Barbarena L, Keske-Soares M, Mota HB. Generalização no tratamento com o /R/ em um caso de desvio fonológico médio-moderado. Rev Soc Bras Fonoaudiol. 2004; 9(4):229-36.

15. Mota HB, Keske-Soares M, Bagetti T, Ceron MI, Melo Filha MG. Análise comparativa da eficiência de três diferentes modelos de terapia fonológica. Pró-Fono. 2007;19(1):67-74.

16. Barbarena L, Keske-Soares M, Mota HB. Generalização baseada nas relações implicacionais obtida pelo modelo "ABAB-Retirada e Provas Múltiplas". Rev Soc Bras Fonoaudiol. 2008;13(2):143-53.

17. Keske-Soares M, Brancalione AR, Marini C, Pagliarin KC, Ceron MI. Eficácia da terapia para desvios fonológicos com diferentes modelos terapêuticos. Pró-Fono. 2008; 20(3):153-8.

18. Keske-Soares M, Mota HB, Pagliarin KC, Ceron MI. Estudos sobre ambientes favoráveis à produção da líquida não-lateral /r/ no tratamento do desvio fonológico. Rev Soc Bras Fonoaudiol. 2007; 12(1):48-54.

19. Mezzomo CL, Ribas LP. Sobre a aquisição das líquidas. In: Lamprecht RR. Aquisição fonológica do português: perfil de desenvolvimento e subsídios para a terapia. Porto Alegre: Artmed, 2004. p.95-112. 